\title{
Faisceaux de rayonnement X ultrabrefs générés par laser
}

\author{
Philippe ZEITOUN, Stéphane SEBBAN, Kim TA PHUOC, Julien GAUTIER et Antoine ROUSSE \\ Laboratoire d'Optique Appliquée, ENSTA-ParisTech, École polytechnique, \\ CNRS, Chemin de la Hunière, 91761 Palaiseau Cedex
}

Le rayonnement $X$ est un des outils les plus puissants pour explorer les propriétés de la matière. Plusieurs générations de très grands instruments, tels que les synchrotrons, puis plus récemment les lasers à électrons libres (LEL) ont été développés, délivrant des faisceaux de rayonnement $X$ aux propriétés de plus en plus remarquables en termes de flux, de durée d'impulsion, d'énergie ou de cohérence. Les LEL sont actuellement les sources de rayonnement $X$ les plus performantes en termes de brillance, mais très peu $d$ 'installations seront disponibles dans le monde du fait de la taille des infrastructures nécessaires et du coût de construction et de fonctionnement. Les lasers intenses ultra-brefs constituent une alternative et la physique de leur interaction avec la matière offre des possibilités uniques pour le développement de sources de rayonnement $X$ qui soient à la fois ultra-brèves et intenses, mais également compactes et à coût modéré, laissant envisager l'installation de ces systèmes au sein de laboratoires de recherche de taille universitaire. Aujourd'hui, grâce aux progrès réalisés dans ce domaine, les plasmas créés par laser permettent de produire des faisceaux innovants de rayonnement $X$. Leur compacité, leur parfaite synchronisation avec un laser intense ou toute source lumineuse ou particulaire générée par le laser, leur brièveté extrême et leur taille de source sub-micrométrique, en font des rayonnements à fort potentiel d'application et de dissémination au niveau académique et sociétal.

\section{Sources $X$ cohérentes créées par laser}

\section{Production de rayonnement harmonique}

En fonction de l'intensité laser focalisée sur une cible, plusieurs processus physiques sont à l'origine de la production de rayonnement $X$. Lors de l'interaction d'un laser intense (intensités supérieures à $10^{14} / \mathrm{cm}^{2}$ ) avec un gaz, du rayonnement harmonique de la longueur d'onde fondamentale du laser se produit lorsqu'un électron, d'abord arraché sous l'effet du champ laser intense, vient se recombiner sur son ion parent après avoir été accéléré dans le continuum. Cette impulsion lumineuse cohérente, composée d'harmoniques impaires de la fréquence fondamentale, est produite dans l'axe du laser générateur. Du rayonnement jusque dans le domaine spectral des $X$ (quelques angströms) est issu de ce processus hautement nonlinéaire : ceci a été démontré au CEA de Saclay au début des années 90. Cette source compacte de rayonnement XUV est particulièrement adaptée pour des études de physique ne requérant pas un flux moyen très important, mais où une résolution temporelle femtoseconde ou attoseconde est nécessaire pour l'étude de dynamiques ultrarapides lors d'expériences de spectroscopie résolue en temps.

\section{Des caractéristiques intéressantes}

La figure 1 montre un spectre typique, obtenuau Laboratoire d'optique appliquée (LOA), sur lequel on peut voir toutes les harmoniques impaires du laser de pompe $(\lambda=800 \mathrm{~nm})$ entre la $43^{\mathrm{e}}(\lambda=800 / 43=$ $18,6 \mathrm{~nm})$ et la $91^{\mathrm{e}}(8,8 \mathrm{~nm})$.

Cette source possède de nombreuses qualités. Le faisceau est très cohérent, polarisé linéairement et possède un bon front d'onde $(\lambda / 7 \mathrm{rms})$ à environ deux fois la limite de diffraction. Les harmoniques sont cohérentes temporellement entre elles. La durée d'impulsion d'une harmonique seule est d'environ $10 \mathrm{fs}$, mais en combinant par exemple plusieurs

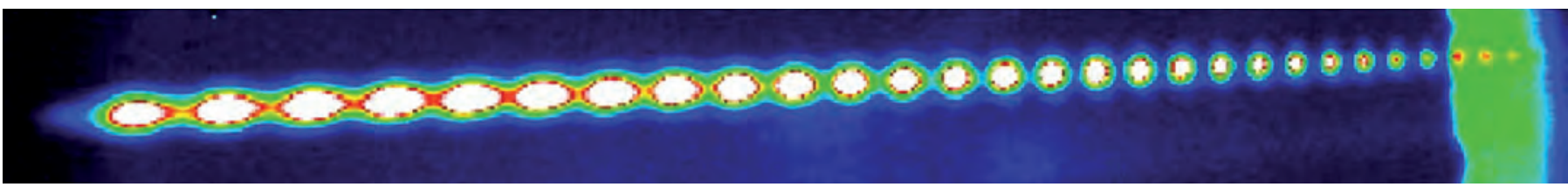

Figure 1. Spectre d'harmoniques (en fausses couleurs) générées dans le néon. Plus l'image est claire, plus le signal est intense. La première harmonique (à gauche) correspond à la 43e harmonique du faisceau laser infrarouge, c'est-à-dire une longueur d'onde d'environ 19 nm. 
harmoniques mises en phase (à l'aide de miroirs spéciaux) la durée d'impulsion peut atteindre 100 as. Les expériences couvrent un large domaine autour de l'imagerie cohérente dans les $X$ faiblement énergétiques (interférométrie, holographie), de mesures d'indice optique, d'études surl'endommagement des miroirs $X$ et d'études de physique du solide telle la dynamique de domaines magnétiques femtosecondes.

\section{Vers la production de flux plus élevés}

Afin de produire des flux $X$ plus élevés que ceux pouvant être produits par la génération d'harmoniques, plusieurs équipes de recherche travaillent à reproduire l'effet d'amplification laser, non plus dans le domaine spectral visible, mais directement dans le domaine des rayons $X$. Le principe est le suivant: un plasma est créé en focalisant un laser sur une cible à l'état solide ou gazeux. Dans certaines conditions de densité et de température, une inversion de population est obtenue entre deux niveaux excités d'une espèce ionique du plasma. L'émission laser $X$ qui suivra est alors la conséquence de cette inversion de population; le rayonnement émis par émission spontanée est amplifié par émission stimulée au cours de sa propagation dans le milieu en inversion de population. Contrairement à une idée courante, l'effet laser ne produit pas intrinsè- quement un faisceau cohérent. C'est l'utilisation d'une cavité optique (deux miroirs placés de part et d'autre du milieu amplificateur selon son plus grand axe) qui, sélectionnant une très faible part de l'émission spontanée du milieu amplificateur, va construire la cohérence au cours de la propagation. II faut en général plusieurs milliers d'aller-retours pour obtenir une forte cohérence. Or la réflectivité des miroirs $\mathrm{X}$ reste faible (le plus souvent 20 à $30 \%$ contre $99,99 \%$ dans le visible), empêchant la mise en place de cavité. Malgré cette limitation, de nombreuses sources lasers $X$ en simple passage (sans cavité), ont été développées à travers le monde. Les plus énergétiques délivrent des impulsions pouvant aller jusqu'à $10 \mathrm{~mJ}$ (mais avec 3 tirs par heure seulement), soit environ 10 millions de fois plus que l'énergie contenue dans une impulsion de synchrotron.

\section{Utilisation d'une configuration oscillateur-amplificateur}

De nombreuses études effectuées à l'aide de systèmes lasers de plus haute cadence ont permis la réalisation de sources laser dans la gamme $30-40 \mathrm{~nm}$ de longueur d'onde. Ces lasers délivrent aujourd'hui des impulsions de l'ordre du $\mu \mathrm{J}$ avec des durées d'impulsion de quelques picosecondes et à une fréquen ce de $10 \mathrm{~Hz}$. Le plasma amplificateur est ici créé en focalisant une impulsion intense (1 J en 30 fs) dans une cellule de gaz. Si ces travaux ont fortement fait progresser la compréhension des processus impliqués dans la réalisation de ce type de sources, dans le début des années 2000 certains points restaient à améliorer (distribution d'énergie dans le faisceau, front d'onde et cohérence spatiale). Dans ce but, le LOA a mis au point, dès le début de l'année 2004, une nouvelle méthode basée sur une géométrie oscillateur-amplificateur (figure 2). Dans cette approche, employée pour les lasers de puissance fonctionnant dans la gamme visible ou infrarouge, le plasma qui est le siège d'une inversion de population n'est plus considéré comme la source à part entière mais comme un amplificateur classique d'une chaîne laser X. II suffit de le coupler à un "oscillateur» X émettant à la même longueur d'onde et ayant de bonnes propriétés de cohérence. Les harmoniques d'ordre élevé se sont naturellement imposées comme l'oscillateur idéal en raison de leur simplicité de mise en œuvre, de la quasi-accordabilité, de la forte cohérence spatiale et d'une polarisation aisément ajustable.

Les résultats de ce type d'expériences furent très convaincants. Le faisceau harmonique incident est amplifié jusqu'à 600 fois, tout en conservant sa polarisation. La cohérence spatiale du faisceau est améliorée ainsi que son front d'onde qui 


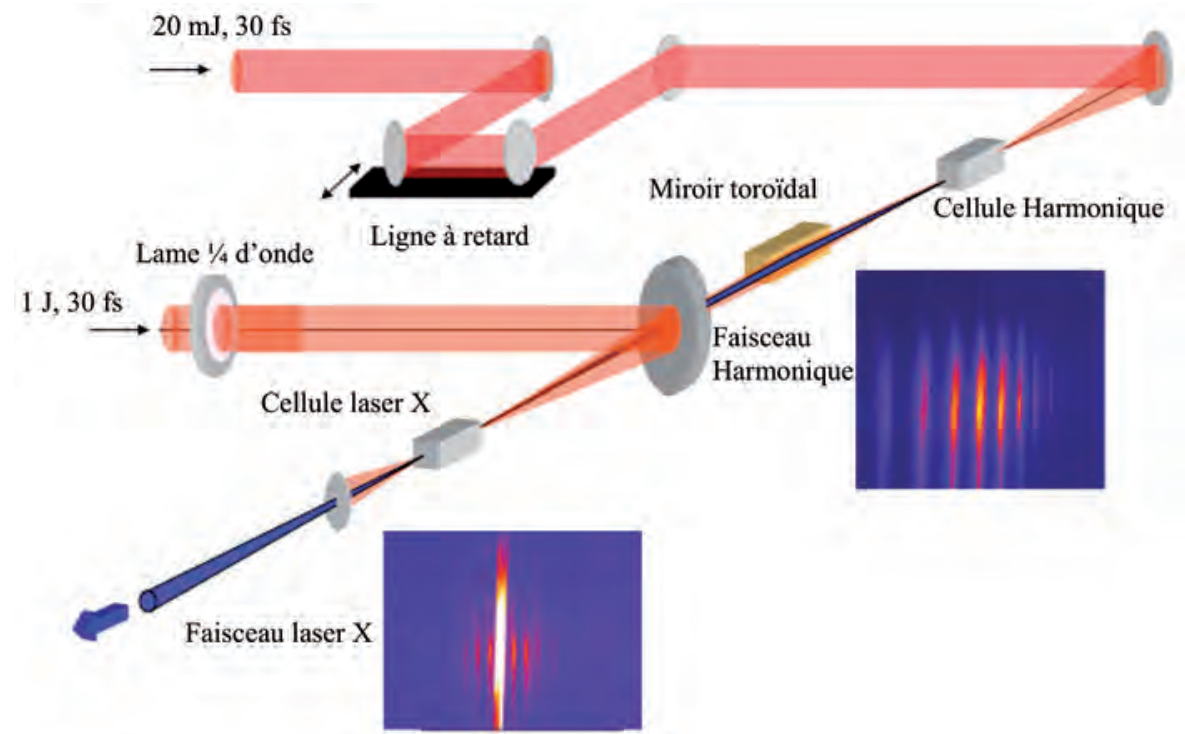

Figure 2. Production d'un faisceau laser à rayon $X$ à partir de l'amplification de rayonnement harmonique dans un plasma créé par laser. Un premier bras du faisceau laser intense est utilisé pour générer des harmoniques. Le miroir toroïdal collecte et focalise ce rayonnement dans un plasma très ionisé créé par le deuxième bras du faisceau laser. La longueur d'onde du rayonnement harmonique correspondant à la longueur d'onde caractéristique de l'inversion de population créée dans le plasma est amplifiée.

atteint la valeur record de $\lambda / 17 \mathrm{rms}$, performance qui est rarement obtenue même sur les lasers infrarouges. Il est probable que l'extension de la technique d'amplification d'harmonique à des plasmas à plus haute densité (comme ceux produits en focalisant l'énergie d'un laser sur une cible massive) permettra d'accrô̂tre l'énergie jusqu'à plusieurs centaines de $\mu \mathrm{J}$ et de réduire la durée des impulsions laser XUV vers la centaine de femtosecondes.

\section{Sources X incohérentes créées par laser}

\section{Interaction à plus haute intensité}

Lors de l'interaction à plus haute intensité $\left(>10^{18} \mathrm{~W} \mathrm{~cm}^{-2}\right)$ entre un laser femtoseconde et un gaz, les processus mis en jeu changent radicalement. Par exemple, des électrons du gaz peuvent être accélérés jusqu'à des énergies fortement relativistes. Ce mécanisme, maintenant bien connu, a été très étudié dans l'objectif de réaliser des accélérateurs compacts de particules par laser. Comme pour les synchrotrons ou les lasers à électrons libres, ces électrons accélérés par laser rayonnement $X$ en les faisant osciller dans électrons en sortie d'accélérateur laser plasma dans des champs magnétiques statiques ; les onduleurs électromagnétiques pour lesquels les électrons oscillent dans un champ laser; et les onduleurs plasma générés par la séparation de charge produite dans un plasma localisé dans le sillage d'une impulsion laser intense se propageant dans un gaz.

Les onduleurs plasmas sont souvent appelés mini-synchrotrons car ils reproduisent, à une échelle millimétrique, le principe d'un synchrotron. Un laser intense, focalisé dans un jet de gaz d'hélium, crée, dans son sillage, une cavité ionique qui joue simultanément les rôles du couple accélérateur - onduleur qui compose habituellement un synchrotron conventionnel. En effet, grâce aux champs électriques intenses existant dans la cavité (qui suit l'impulsion laser), les électrons du plasma qui y sont piégés sont accélérés jusqu'à plusieurs centaines de mégaélectronvolts ( $\mathrm{MeV}$ ) et oscillent avec une période de l'ordre d'une centaine de microns et avec une amplitude de l'ordre du micromètre. La conséquence de ce mouvement est l'émission d'un faisceau de rayonnement $X$ intense que I'on appelle rayonnement bétatron (figure 4). Ce rayonnement a été mis en évidence pour peuvent être utilisés pour générer du des onduleurs (figure 3). Différents types d'onduleurs ont été étudiés par les équipes de recherche : les onduleurs à aimants permanents qui font osciller les
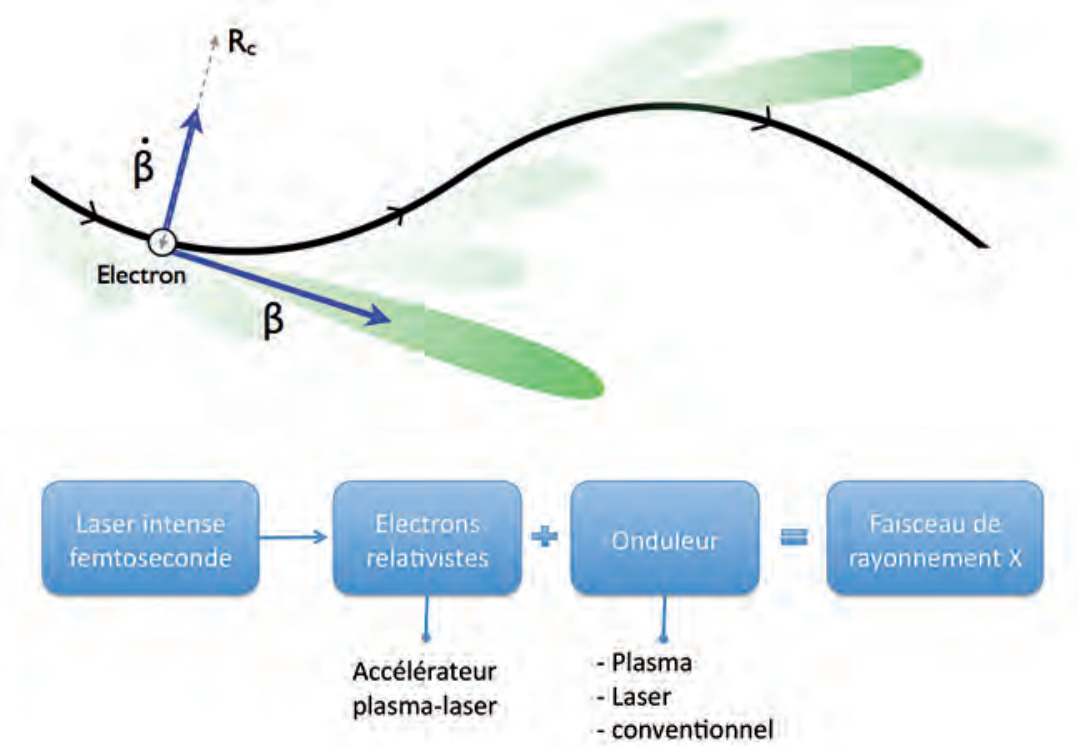

Figure 3. Un électron relativiste accéléré dans un plasma et dont la trajectoire effectue des oscillations rayonne un faisceau collimaté vers l'avant dans le domaine spectral $X$. Plusieurs types d'onduleurs ont été étudiés par les physiciens : les onduleurs conventionnels formés d'aimants permanents successifs ; les onduleurs plasmas créés par une séparation de charge dans le milieu très ionisé ; les onduleurs lasers correspondant aux champs électromagnétiques oscillant d'un laser. 
Les atouts des rayons $X$ ultra-rapides

Les rayons $X$ possèdent trois qualités majeures qui les différencient de la lumière visible ou ultraviolette.

Les rayons $X n^{\prime}$ interagissent pas avec la matière comme le fait du rayonnement de plus faible énergie. La lumière visible tend à exciter ou casser des liaisons chimiques entre atomes d'une molécule. Au contraire, pour les rayons $X$, l'interaction se fait directement avec les électrons de cœur des atomes offrant une très grande sélectivité spectrale vis-à-vis des constituants élémentaires de l'échantillon (et non plus des molécules). Sur synchrotron, cette propriété est utilisée depuis plusieurs décennies pour effectuer des analyses ultrafines. Ce n'est qu'avec l'apparition très récente de sources ultra-intenses de rayonnement $X$ que I'on peut utiliser cette sélectivité spectrale pour porter la matière dans un état très particulier, dit fortement dégénéré, présent au cœur de planètes géantes comme Jupiter.

La seconde propriété est la très petite limite de diffraction. En simplifiant le concept, on peut dire qu'il n'est pas possible d'imager un objet ou de focaliser de la lumière sur une dimension inférieure à la longueur d'onde. En passant du rayonnement visible aux rayons $X$, les dimensions caractéristiques passent du micromètre $\left(10^{-6} \mathrm{~m}\right)$ au nanomètre $\left(10^{-9} \mathrm{~m}\right)$ voire au picomètre $\left(10^{-12} \mathrm{~m}\right)$. Un monde totalement nouveau s'ouvre aux chercheurs utilisant des rayons $X$. Associé à la sélectivité spectrale, le pouvoir résolvant des rayons $X$ peut devenir un outil extraordinaire pour l'analyse ou l'excitation de la matière dans des conditions encore inconnues.
La possibilité de focaliser le rayonnement sur des taches nanométriques ouvre bien évidemment la voie à la physique de l'interaction non-linéaire entre des rayons $X$ et la matière ; ce type $d^{\prime}$ expérience était jusqu'à très récemment limité aux lasers visibles ou infrarouges.

La troisième propriété, actuellement mise à profit uniquement sur les sources de rayons $X$ produites par laser, provient de la limite de Fourrier. Celle-ci peut se comprendre comme une extension du théorème $d^{\prime} H$ Heisenberg $\Delta E \times \Delta t \geq h / 2 \pi$, où $\Delta E$ est le spectre en énergie, $\Delta t$ la durée d'impulsion et $h$ vaut 6,62 $\times 10^{-34} \mathrm{~J} \mathrm{~s}$. L'ensemble du spectre visible est limité à 0,5 eV environ ce qui correspond à une durée d'impulsion minimale de 2,7 fs (avec $1 \mathrm{fs}=10^{-15} \mathrm{~s}$ ). Pour les sources produites par laser, le spectre des rayons $X$ peut couvrir jusqu'à $10 \mathrm{keV}$ ce qui correspond à une durée minimale de $130 \mathrm{zs}\left(1 \mathrm{zs}=10^{-21} \mathrm{~s}\right)$. Pour atteindre la limite de Fourier $(\Delta \mathrm{E} \times \Delta t=$ $h / 2 \pi)$, l'ensemble des photons doivent être émis de manière cohérente durant l'impulsion. Ce phénomène a été démonté depuis une décade environ dans la gamme spectrale des rayons $X$ mous donc avec un spectre couvrant environ $20 \mathrm{eV}$ et une durée d'impulsion mesurée à 100 as ( 1 as $=10^{-18} \mathrm{~s}$ ). Bien que toutes les sources $X$ produites par laser ne soient pas limitées par Fourier, les durées d'impulsion sont généralement de l'ordre de quelques femtosecondes à la picoseconde, ce qui reste particulièrement court comparé aux impulsions produites sur synchrotron (dizaines de picosecondes).

\section{Cavité ionique}

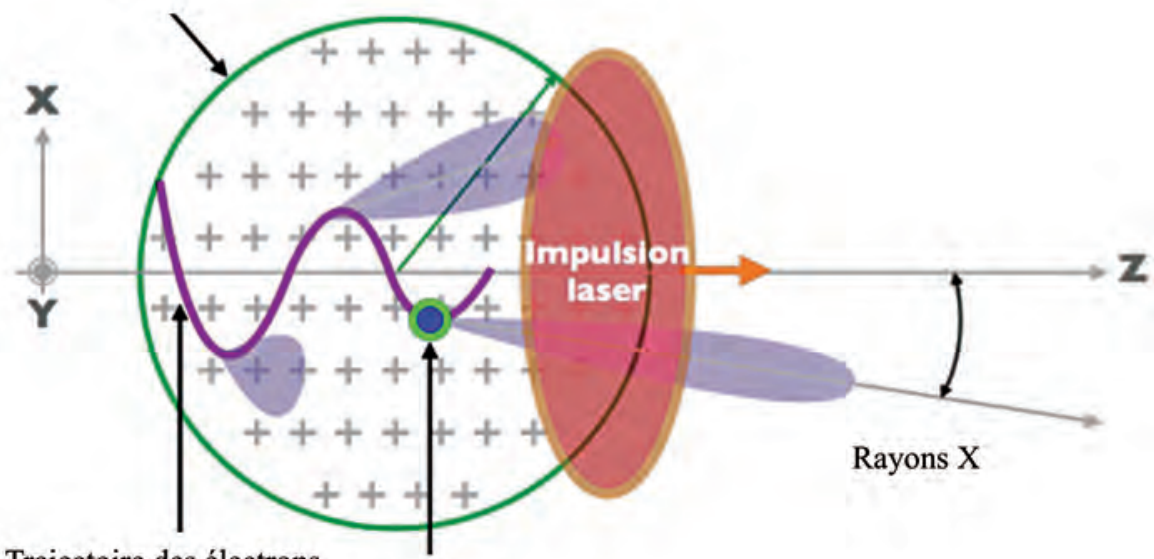

Trajectoire des électrons

\section{Electrons}

Figure 4. Le rayonnement $X$ bétatron est produit dans le sillage $\mathrm{d}^{\prime}$ une impulsion laser femtoseconde intense se propageant dans un gaz. La bulle de plasma créée par le laser est une cavité ionique entourée de charges négatives. Elle joue le rôle d'accélérateur et d'onduleur car les champs longitudinaux et transversaux issus de la séparation de charge accélèrent et font osciller les électrons pour émettre le faisceau de rayonnement $X$.

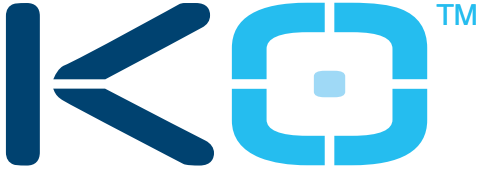

KNIGHT OPTICAL
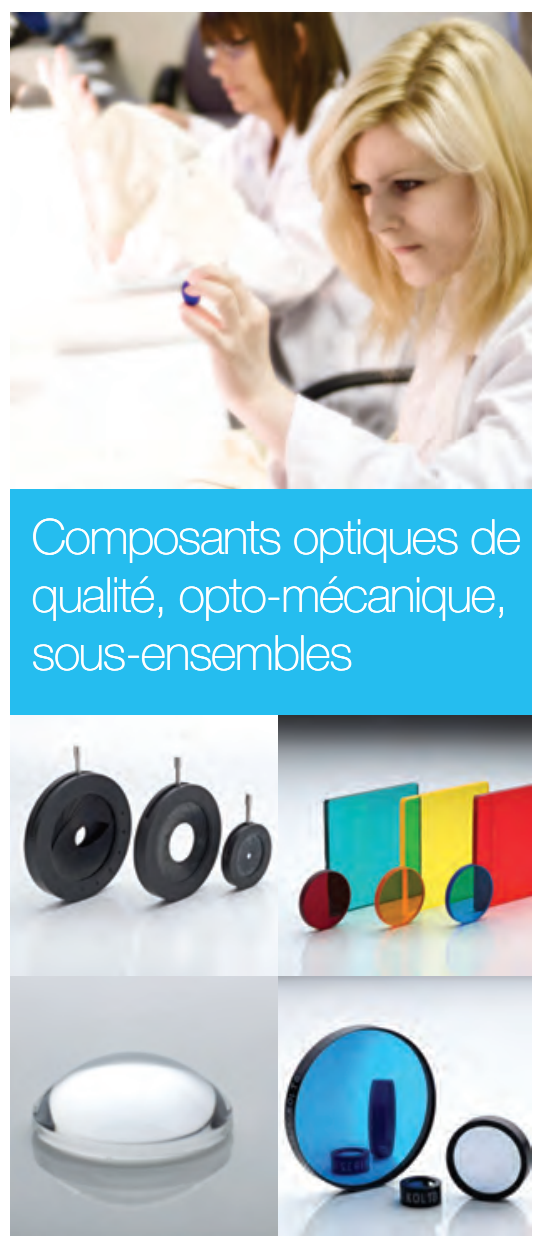

Notre équipe de
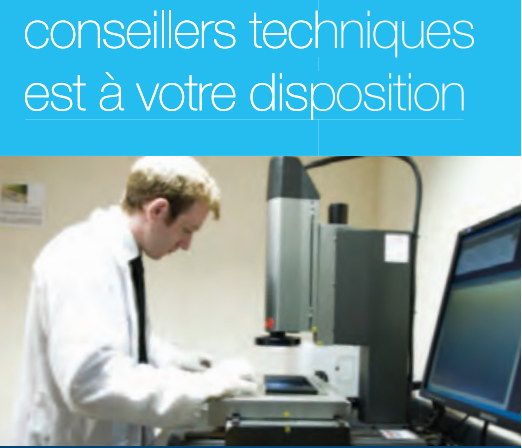

CONTACT

Tél. : +44 1622850614

BertrandMarcillaud@knightoptical.co.uk

knightoptical.com 
la première fois au LOA en 2004. Depuis, de nombreux laboratoires s'intéressent à cette source et la développent.

Le montage expérimental, simple et compact, rend cette source particulièrement attractive pour les utilisateurs. Dans le cas du LOA, le laser de la Salle Jaune $(1,5 \mathrm{~J}, 30 \mathrm{fs}, 10 \mathrm{~Hz})$ est focalisé dans un jet supersonique d'hélium de longueur millimétrique (figure 5). Un aimant est placé à I'arrière du jet et est utilisé pour séparer le rayonnement $X$ des électrons accélérés.

\section{Le rayonnement bétatron}

Le faisceau de rayonnement bétatron est maintenant bien caractérisé. L'émission est de type "spectre blanc », caractéristique d'une fonction synchrotron. Il est continu entre quelques eV et plusieurs dizaines de keV (figure 6). Le faisceau est faiblement divergent (environ $10 \mathrm{mrad}$ ) avec une distribution quasi-gaussienne. La taille de source est d'environ $1 \mu \mathrm{m}$ et la durée d'impulsion a été estimée à l'aide de simulations numériques à moins de $20 \mathrm{fs}$. L'ensemble de ces paramètres en font une source particulièrement attractive pour les utilisateurs en physique des plasmas (radiographie ultra-rapide, spectroscopie d'absorption), en imagerie biologique (radiographie, microscopie) et en physique du solide (étude de la dynamique de structure comme les transitions de phase ultra-rapide).

Le rayonnement bétatron n'est qu'au début de son histoire. De nombreuses améliorations sont attendues, sur le nombre de photons par impulsion (en utilisant un laser plus énergétique), sur la puissance moyenne (en augmentant le taux de répétition du laser) ou sur l'énergie maximale des photons. Ce dernier point représente un enjeu majeur car, dès l'accès à un flux de photons suffisant aux

Filtre Be

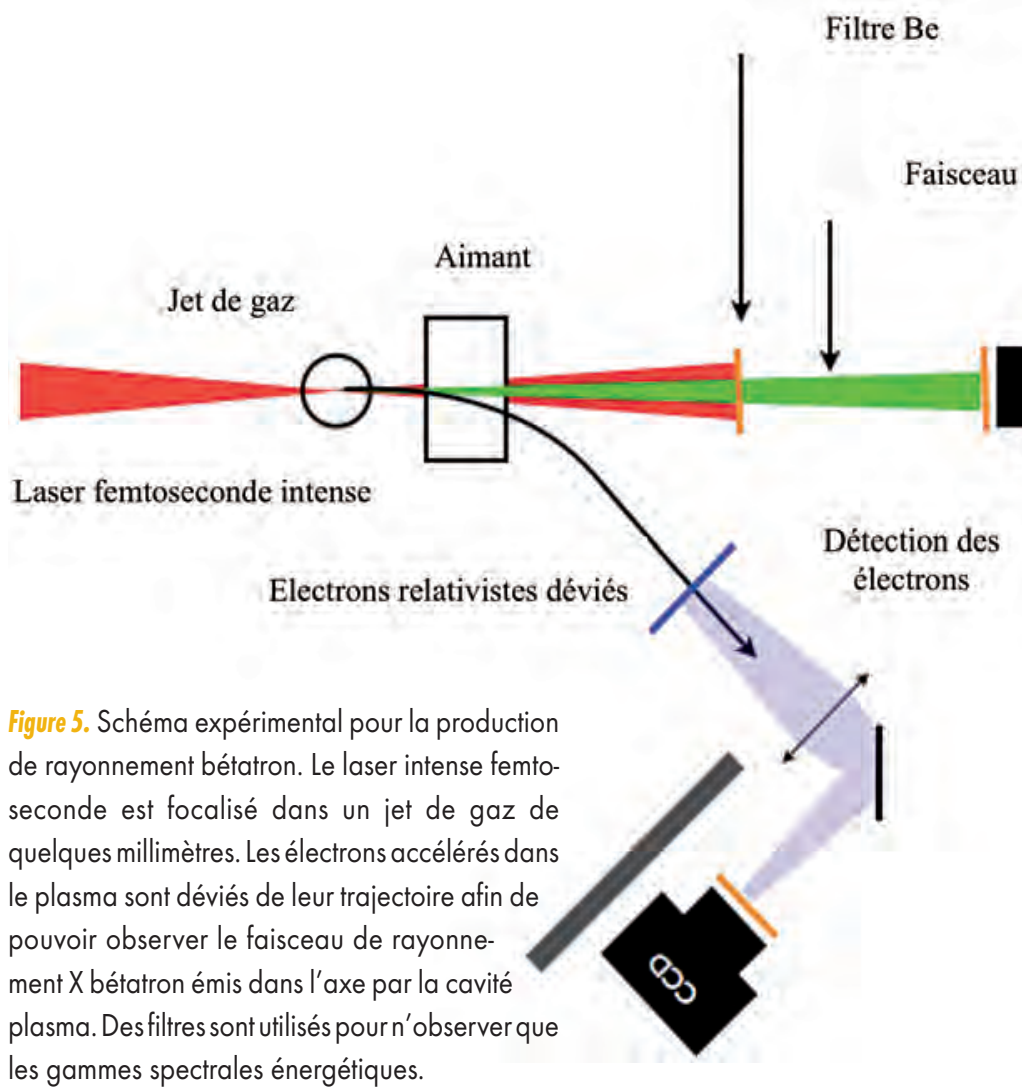

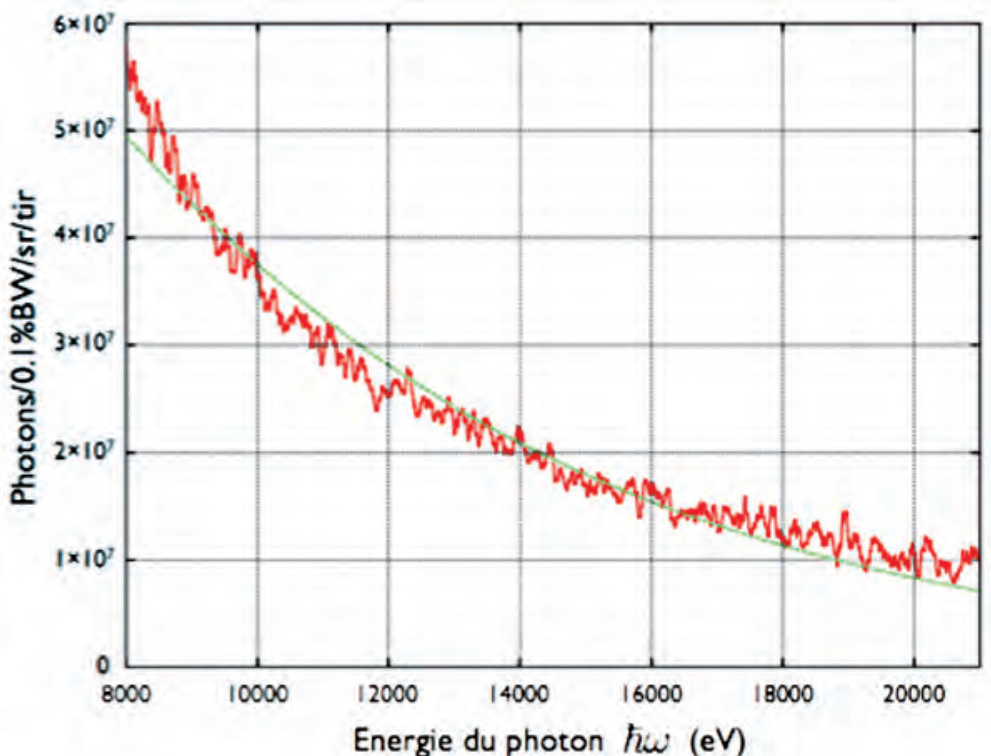

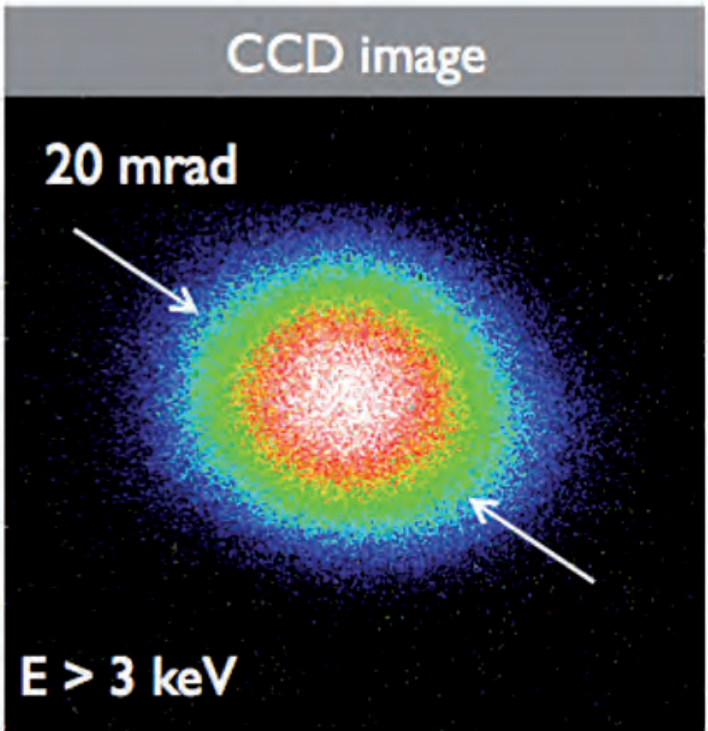

Figure 6. La signature spectrale du rayonnement bétatron est une fonction du type synchrotron. Lors des expériences effectuées à l'INRS (Québec), du rayonnement au-delà de $10 \mathrm{keV}$ a été obtenu. Le faisceau est collimaté sur 20 mrad autour des longueurs d'onde de $3 \mathrm{keV}$. 
Applications des X ultrarapides

Le champ d'applications des sources de rayons $X$ est très vaste. Un synchrotron par exemple, source de rayons $X$ de référence, accueille en moyenne un millier d'utilisateurs par an. Ceux-ci viennent de tous les horizons, médecine, biologie, chimie, physique et même arts (étude d'œuvres d'art). Les sources de rayonnement $X$ produites par laser n'ont pas atteint cette maturité, qui imposerait par ailleurs des moyens humains et techniques bien supérieurs à ceux qui sont disponibles dans cette communauté scientifique actuellement. Par ailleurs, ces sources doivent très certainement encore trouver leur «niche» scientifique. Quels sont les points forts des sources de rayons $X$ créées par laser? Les durées $d^{\prime} i m$ pulsions ultra-brèves (picoseconde jusqu'à attoseconde), la brillance, la compacité et leur synchronisation parfaite avec le faisceau laser intense femtoseconde sont les atouts majeurs. Les expériences nécessitant un grand nombre de photons comme les effets non-linéaires dans le domaine des rayons $X$, l'imagerie haute résolution, l'imagerie de milieu émissif comme un plasma, l'étude des dommages photoinduits, la création de plasmas, ou bien les expériences d'étude d'effets dynamiques dans la matière comme la dynamique ultrabrève des électrons, des atomes, des molécules ou des structures sont particulièrement bien adaptées. Les sources $X$ produites par laser étant intrinsèquement synchronisées au laser femtoseconde intense, les expériences de spectroscopie ultrarapide du type pompe-sonde où les faisceaux d'excitation (pompe) et de sonde d'un échantillon peuvent être de nature très différentes, par exemple infrarouge- $X$, ou $X-X$, ou encore $X$-particules énergétiques deviennent accessibles.

longueurs d'onde de quelques dizaines de keV, de très nombreuses applications médicales apparaissent, comme par exemple l'imagerie de tumeurs cancéreuses avec une haute résolution spatiale.

\section{Vers de plus hautes énergies}

Comment atteindre cette gamme d'énergie ? Plusieurs paramètres de l'interaction peuvent être utilisés. L'expression donnant l'énergie caractéristique du rayonnement bétatron dépend en effet du produit entre la densité du plasma dans lequel est focalisé le laser intense, l'amplitude d'oscillation des électrons accélérés dans l'onduleur plasma, et le carré du facteur de Lorentz qui est une quantité représentant l'énergie des électrons accélérés. Chaque paramètre influe sur les propriétés générales du faisceau de rayonnement X. Augmenter les amplitudes d'oscillation produira un faisceau plus divergent, alors que l'augmentation des énergies des électrons le rendra plus collimaté mais nécessitera des onduleurs plasma de plus grandes longueurs ce qui est actuellement difficile à produire.

Le développement de la source de rayonnement bétatron va suivre celui des lasers, qui sont de plus en plus intenses. Plusieurs sont en construction au LOA et ailleurs. Avec un laser de classe multi-Pettawatt, bientôt accessible avec le projet d'Équipements d'excellence CILEX récemment sélectionné dans le cadre des projets d'Investissements d'avenir, un faisceau de rayonnement $X$ collimaté dans un angle de quelques mrad dans la gamme de longueur d'onde correspondant à plusieurs centaines de keV avec plus de $10^{8}$ photons/tirs et $0,1 \%$ de largeur spectrale est par exemple attendu.

\section{Des applications en constante évolution}

En moins de dix ans, le développement des sources $X$ femtosecondes créées par laser intense a très fortement progressé. Alors que de nouveaux processus d'interaction ont été découverts, l'optimisation des caractéristiques spatio-temporelles des sources les plus abouties et le développement de la métrologie $X$ permettent maintenant à des utilisateurs extérieurs provenant de communautés scientifiques diverses de les utiliser. Quelques lignes de lumière exclusivement réservées aux utilisateurs sont maintenant en opération dans le monde, en particulier pour les études d'imagerie haute résolution spatiale et temporelle. Le meilleur reste probablement à venir. Les avènements, à très court terme, à la fois des lasers femtosecondes ultra-intenses (intensité supérieure à $10^{22} \mathrm{~W} / \mathrm{cm}^{2}$ ) de basse cadence et des lasers intenses de haute cadence (intensité supérieure à $10^{18} \mathrm{~W} / \mathrm{cm}^{2}$ ) vont considérablement ouvrir le champ des applications des sources de rayonnement $\mathrm{X}$ produites par laser.

\section{Plateforme OSICS Créez vos Bancs de Tests}
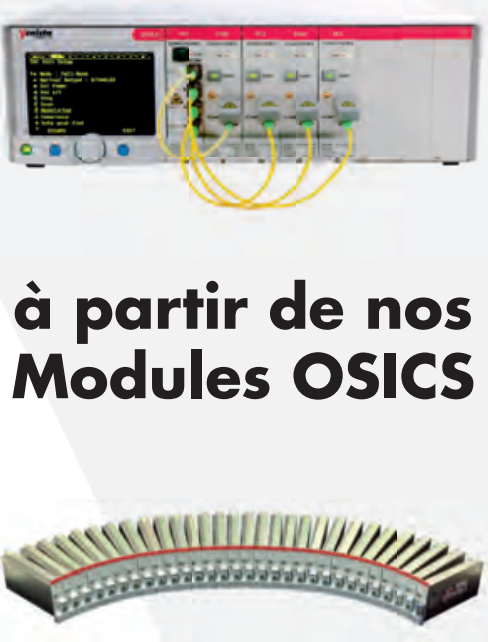

T100 : Lasers accordables

DFB : Lasers de transmission

TLS : Transmission cohérente

SLD : SLED

SWT : Commutateurs

ATN : Atténuateurs

BKR : Réflecteurs

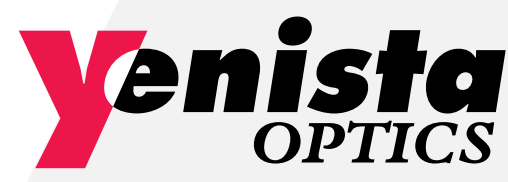
Tél. : +33 (0)296483716 sales-emea@yenista.com www.yenista.com

